\title{
Fenomenología de los Recuerdos Autobiográficos en el Espectro Depresivo: Una Revisión
}

\author{
María Lolich ${ }^{1}$
}

\section{Artículo}

Material original autorizado para la publicación en la revista Psicodebate. Facultad de Ciencias Sociales. Universidad de Palermo.

Recibido 01-07-2014 | Aceptado 05-08-2014

\section{Resumen}

La experiencia de rememoración es distintiva de los recuerdos autobiográficos. Si bien memoria y afectividad se encuentran íntimamente relacionadas, son recientes los estudios que buscan indagar en los perfiles fenomenológicos de evocación durante la ocurrencia de alteraciones afectivas. El presente trabajo expone una revisión de las características fenomenológicas de los recuerdos autobiográficos en presencia de alteraciones del espectro depresivo. Se llevo a cabo una revisión bibliográfica en más de 10 bases de datos (Academic Search Complete, Cambridge Journals Online, Directory of Open Access Journals, PsycArticles, PsycINFO, PUBMED, SCOPUS, SwetsWise, Taylor, Francis Online y Wiley Online Library) de aquellos artículos que abordaron la fenomenología de los recuerdos autobiográficos en individuos con alteraciones depresivas publicados durante los años 2000-2014. Fueron incluidos para el análisis un total de 13 publicaciones. Los estudios concluyen que durante la presencia de sintomatología depresiva clínicamente significativa los recuerdos de valencia positiva son evocados desde una perspectiva de tercera persona, con bajos niveles de intensidad emocional y escasa vivacidad. Por otro lado, los recuerdos negativos de tipo intrusivo resultan altamente vívidos y emocionalmente intensos, acompañados por distintos componentes sensoriales. Los resultados preliminares sugieren que los atributos fenomenológicos, tanto de los recuerdos positivos como negativos, se encontrarían relacionados con la aparición y evolución a corto plazo de los cuadros depresivos. Además, una modalidad de evocación de tipo generalizada, en contraposición con una evocación específica, se encontró asociada con la presencia de sintomatología afectiva. Considerando los

1 Centro de Investigación en Neurociencias y Neuropsicología Clínica, Facultad de Ciencias Sociales, Universidad de Palermo, Buenos Aires, Argentina; Iolichm@yahoo.com.ar 
vínculos entre el sí mismo y la memoria autobiográfica y, dado que los modelos psicoterapéuticos requieren que los individuos exploren su pasado personal, el promover un procesamiento específico de los recuerdos autobiográficos constituye un recurso clínico de relevancia. Teniendo en cuenta la etapa preliminar de estas líneas de investigación, es necesario continuar la exploración de la fenomenología de los recuerdos durante la presencia de trastornos afectivos.

Palabras Clave: Memoria autobiográfica - Fenomenología - Depresión Especificación - Emoción.

\section{Abstract}

Autobiographical memories are characterized by their recollection experience. While memory and emotion are closely related, studies which aimed to investigate memories' phenomenological profiles during affective disorders are quite recent. This paper presents a review of autobiographical memories' phenomenological characteristics during depressive spectrum disorders. A literature review was conducted in more than 10 databases: Academic Search Complete, Cambridge Journals Online, Directory of Open Access Journals, PsycArticles, PsycINFO, PUBMED, SCOPUS, SwetsWise, Taylor and Francis Online Wiley Online Library; of those articles published during the years 2000-2014 that addressed autobiographical memories phenomenology in individuals with depressive disorders. A total of 13 articles were included for analysis. Current studies conclude that, in the presence of depressive symptoms, positively valence memories are evoked predominantly from an observer perspective, with poor emotional intensity and vitality levels. Furthermore, intrusive negative memories are highly vivid and emotionally intense, accompanied by different sensory components. Preliminary results suggest that phenomenological features, both in positive and negative memories, would be related to the onset and short-term evolution of depressive symptoms. In addition, an overgeneral evocation style, as opposed to a specific evocation, was found associated with the presence of affective symptoms. Considering the links between self and autobiographical memory, and since psychotherapeutic models require individuals to explore their personal past, promoting autobiographical memories specific processing is an important clinical resource. Given the preliminary stage of research, further exploration of memories phenomenology during the presence of affective disorders is warranted.

Keywords: Autobiographical memory - Phenomenology - Depression Specificity - Emotion. 
Desde las ciencias cognitivas se entiende a la memoria autobiográfica (MA) como un sistema de procesamiento de información personal. La misma posibilita la conservación y evocación de experiencias significativas del pasado por períodos prolongados (Haringsma, Spinhoven, Engels, \& Van der Leeden, 2010; Tulving, 2002). Durante los años setenta su abordaje experimental se realizó, casi con exclusividad, por medio de pruebas de aprendizaje verbal (Cohen, 2008). Hoy en día se pretende una aproximación más ecológica, por lo que fueron incorporados en los programas cognitivos de investigación el estudio de sus propiedades fenomenológicas y su relación con el auto-concepto (Tulving, 1985; Tulving, 2002).

Rubin y Siegler (2004) describen a la MA como un sistema multimodal, caracterizado por la operación conjunta de componentes sensoriales, espaciotemporales, narrativos y afectivos. Otros componentes fenomenológicos que pueden manifestarse durante la evocación de recuerdos autobiográficos son la perspectiva visual, la sensación de viajar atrás en el tiempo (conciencia autonoética) y su expresión contraria, la sensación de actualidad (Newby \& Moulds, 2011b; Sutin \& Robins, 2007; Tulving, 2002).

En los últimos años, además, comenzó a tomar fuerza la distinción entre los recuerdos autobiográficos de tipo específico (RAE) versus los recuerdos autobiográficos de tipo generalizados. Los RAE dan cuenta de eventos ocurridos en un espacio y en un tiempo delimitados mientras que los recuerdos generalizados hacen a una suma imprecisa de eventos similares. El formato representacional de los RAE asume características multimodales (e.g. visuales, temporales, afectivas) y el de los recuerdos generalizados de tipo proposicional (Rubin \& Siegler, 2004; Joormann, Siemer, \& Gotlib, 2007; Williams et al., 2007). Además, los RAE participan de las rutinas cotidianas, son utilizados en las tomas de decisiones, contribuyen a la construcción de la identidad y emergen como referentes en los procesos de cognición social (Conway, 2005; Conway \& Pleydell-Pearce, 2000; Sutin \& Robins, 2007).

Estudios en psicología cognitiva (Patel et al., 2007; Williams et al., 2007) proponen la aparición de cambios distintivos en el funcionamiento mnémico durante la presencia de sintomatología depresiva. La MA desempeña un rol de consideración en el inicio, mantenimiento y evolución de los trastornos afectivos por medio de sesgos de procesamiento congruentes con el estado de ánimo (Rottenberg, Joormann, Brozovich, \& Gotlib, 2005). Por ejemplo, algunos recuerdos pueden favorecer un descenso del tono afectivo, sin importar si estos son de valencia negativa (Singer \& Salovey, 1988; Williams, Watts, MacLeod, \& Mathews, 1988; Werner-Seidler \& Moulds, 2011a) o positiva (Gillihan, Kessler, \& Farah, 2007). También se ha reportado que la capacidad cognitiva para acceder a RAE se encuentra alterada (Scott \& Williams, 1988). Se observa así un incremento en la tendencia a evocar recuerdos generalizados (Williams, 1996). Por tanto, la 
presencia de sintomatología depresiva clínicamente significativa no sólo incide en una mayor evocación de recuerdos negativos sino que también afecta las vías de acceso a los atributos fenomenológicos de los RAE (Williams et al., 2007).

Dichas dificultades serían el resultado de estrategias que buscan regular el tono anímico. Se pretendería, infructuosamente, evitar la posibilidad de reexperimentar de forma detallada las vivencias de un pasado que resulta disonante en el presente (Williams et al., 2007). Sin embargo, los modelos clínicos consideran que el promover la especificación de los atributos fenomenológicos de los recuerdos puede resultar un valioso recurso psicoterapéutico (e.g. Brewin et al., 2009; Segal, Williams, \& Teasdale, 2002). Un procesamiento específico de la información posibilita que ella sea asimilada a las redes mnémicas correspondientes, disminuyéndose los niveles de afectividad disruptivos o incentivándose, en cambio, afectos reconfortantes (Hermans, Defranc, Raes, Williams, \& Eelen, 2005). En consonancia, distintas estrategias clínicas buscan promover el procesamiento consciente y específico de la información, tal como sucede con las técnicas de mindfulness (Bishop et al., 2004; Brown, Ryan, \& Creswell, 2007), imaginería guiada u otras técnicas de imaginería en general (Kandris \& Moulds, 2008).

Actualmente los proyectos de investigación en curso buscan abordar con mayor profundidad los vínculos entre los trastornos mentales y el perfil experiencial de los RAE. Los estudios suelen valerse del Test de Memoria Autobiográfica (Autobiographical Memory Test o AMT por sus siglas en inglés) para evaluar el grado de sobregeneralización o especificidad de los recuerdos (Williams \& Broadbent, 1986). Dicha prueba promueve la evocación de recuerdos autobiográficos en respuesta a una serie de palabras claves de valencia positiva, negativa o neutra (Rottenberg et al., 2005; Zinbarg \& Rekart, 2006). Transcurridos 60 segundos se contabilizan la cantidad y tipo de recuerdos evocados (Williams et al., 2007). Complementariamente, se suelen administrar otras pruebas que indagan en los atributos fenomenológicos (Rubin, Schrauf, \& Greenberg, 2003; Sutin \& Robins, 2007). Las mismas evalúan el grado de vivacidad, de coherencia narrativa, el tipo de perspectiva visual, los componentes sensoriales, la intensidad emocional, la sensación de actualidad o la conciencia autonoética. Los resultados alcanzados hasta el momento reportan que el perfil fenomenológico de los RAE muestra asociaciones significativas con los rasgos de personalidad, la tipología del recuerdo, distintos trastornos psicopatológicos, el género y la edad (Sutin \& Robins, 2007). Sin embargo, sólo algunos estudios han abordado de forma particular las características fenomenológicas de los RAE durante la presencia de alteraciones afectivas.

Dado que la aproximación sistemática de los atributos implicados en la experiencia de evocación es un tópico relativamente reciente, el presente trabajo 
busca realizar una revisión de aquellos estudios que evaluaron los componentes fenomenológicos de los recuerdos autobiográficos evocados por individuos con alteraciones del espectro depresivo.

\section{Método}

Fue realizada una revisión bibliográfica en Academic Search Complete, Cambridge Journals Online, Directory of Open Access Journals, PsycArticles, PsycINFO, PUBMED, SCOPUS, SwetsWise, Taylor, Francis Online y Wiley Online Library. Fueron incluidos sólo aquellos artículos publicados durante el 2000-2014, en idioma inglés o español, que resultaron de la combinación de los términos autobiographical memory (memoria autobiográfica), episodic memory (memoria episódica), self-defining memory (recuerdo auto-descriptivo) y personal memory (recuerdo personal) con las siguientes palabras clave: vividness (vivacidad), visual perspective (perspectiva visual), sensory details (detalles sensoriales), emotion (emoción), coherence (coherencia), accessibility (accesibilidad), autonoetic consciousness (conciencia autonoética), phenomenology (fenomenología), experience (experiencia), reliving (revivir), re-experience (reexperimentar), depressive symptoms (síntomas depresivos), dysthymia (distimia), y depression (depresión).

\section{Resultados}

Un total de 13 artículos cumplieron con los criterios de búsqueda. Entre ellos, siete abordaron los componentes fenomenológicos de acuerdo con la valencia positiva o negativa de los RAE mientras que los seis artículos restantes exploraron la fenomenología de los RAE de tipo intrusivo. Participaron muestras de individuos con depresión mayor, recuperados de episodios depresivos, individuos disfóricos, individuos con sintomatología depresiva clínicamente significativa no especificada e individuos control. Para la evaluación de los componentes fenomenológicos y sintomatología afectiva se utilizaron distintas escalas, inventarios y entrevistas (ver tablas 1, 2 y 3 para más detalles relativos al número de individuos en los distintos grupos muestrales e instrumentos utilizados).

Teniendo en cuenta los componentes abordados por los distintos estudios, la exposición de los resultados se desenvuelve de acuerdo a los siguientes ejes: I) valencia afectiva (tabla 1), II) conciencia autonoética y perspectiva visual (tabla 2), y III) recuerdos intrusivos (tabla 3). 


\section{Valencia Afectiva}

Siguiendo la hipótesis de que la evocación de RAE continúa presentando alteraciones una vez finalizado el episodio afectivo, Werner-Seidler \& Moulds (2011a) exploraron las características fenomenológicas de los RAE de valencia positiva y negativa en individuos recuperados de un episodio depresivo en comparación a individuos control. Los participantes evocaron RAE bajo la influencia de distintos estados anímicos (ver tabla 1). Los grupos no difirieron significativamente en sus recuerdos al encontrarse en un estado de ánimo neutro, pero al ser inducidos a un estado de ánimo negativo, aquellos individuos con antecedentes afectivos recordaron con menor vivacidad a los RAE de valencia positiva (Werner-Seidler \& Moulds, 2011a).

En un segundo estudio, luego de inducir al grupo de individuos con antecedentes depresivos e individuos control a un estado de ánimo negativo, los mismos indicaron el grado de vivacidad, perspectiva visual y accesibilidad de los RAE de valencia positiva. Solo la dimensión vivacidad resultó significativamente distinta en ambos grupos, siendo los RAE del grupo con antecedentes depresivos menos vívidos (Werner-Seidler \& Moulds, 2011a).

Por otra parte, Sutin y Gillath (2009) exploraron las características de los RAE con relación a distintos estilos de apego y presencia de sintomatología depresiva. Alumnos universitarios evocaron RAE vinculados con situaciones románticas. Los participantes completaron escalas autoadministrables para evaluar la coherencia de los RAE, su intensidad emocional, el estilo de apego y grado de sintomatología afectiva. La coherencia de los RAE y la intensidad emocional se encontraron asociados negativamente con un estilo de apego evitativo y con la presencia de sintomatología depresiva clínicamente significativa (tabla 1). Los autores sugieren que el malestar afectivo en aquellos individuos con un estilo de apego evitativo procede de recuerdos autobiográficos incoherentes con bajos niveles de intensidad emocional (Sutin \& Gillath, 2009).

Por fuera del paradigma que emplea palabras clave para estimular la evocación, Rottenberg et al. (2005) utilizaron entrevistas ideográficas y pruebas psicológicas del estado de ánimo a individuos con depresión. La entrevista ideográfica se focaliza en RAE de los momentos más y menos agradables de la vida de acuerdo con la perspectiva de los participantes. Cada RAE fue puntuado por los investigadores de acuerdo con el nivel de detalle contextual referido e intensidad emocional. Un año más tarde, los niveles de sintomatología depresiva fueron reevaluados. El grado de intensidad emocional correlacionó negativamente con los niveles de sintomatología depresiva: cuanto más baja resultó la intensidad emocional en el tiempo basal, más alta fue la severidad de sintomatología 
depresiva encontrada durante el seguimiento. La intensidad emocional y la cantidad de detalles sensoriales se encontraron moderadamente asociados. El estudio concluye que un bajo nivel de intensidad emocional incrementa la vulnerabilidad individual a desarrollar sintomatología afectiva a mediano plazo (Rottenberg et al., 2005).

\section{Conciencia Autonoética y Perspectiva Visual}

Tulving $(1985,2002)$ introdujo el concepto de conciencia autonoética con relación a la sensación de viajar atrás en el tiempo al recordar episodios del pasado personal. Asimismo, las imágenes que acompañan a la evocación de los RAE pueden ser reexperimentadas desde distintas perspectivas visuales. Los detalles pueden ser evocados desde la perspectiva original desde la que se vivenció el evento (perspectiva de primera persona) o desde una posición externa (perspectiva de tercera persona).

Lemogne et al. (2006) evaluaron los RAE en personas que transitaban un episodio depresivo mayor con relación al grado de especificidad, la valencia emocional, la conciencia autonoética y la perspectiva visual (tabla 2). Al comparar la presentación de dichos componentes con un grupo control, encontraron que los RAE de valencia positiva en el grupo clínico presentaron un menor grado de conciencia autonoética. Los hallazgos también indicaron que la perspectiva visual de evocación más utilizada por el grupo clínico fue de tercera persona. 
Tabla 1.

Valencia de los Recuerdos Autobiográficos.

\begin{tabular}{|c|c|c|c|c|}
\hline Estudio & $\begin{array}{l}\text { Características } \\
\text { de la Muestra }\end{array}$ & Instrumentos & $\begin{array}{l}\text { Componentes } \\
\text { fenomenológi- } \\
\text { cos bajo estudio }\end{array}$ & $\begin{array}{l}\text { Hallazgos } \\
\text { Principales }\end{array}$ \\
\hline 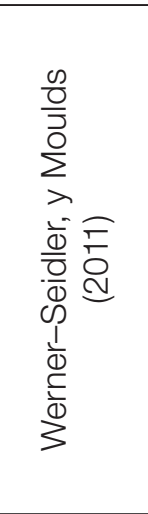 & $\begin{array}{l}\text { Estudio 1: } \\
\text { Individuos con } \\
\text { antecedentes } \\
\text { depresivos }(n= \\
87) \text { e individuos } \\
\text { control }(n=49) \\
\text { Estudio 2: } \\
\text { Individuos con } \\
\text { antecedentes ( } n \\
=50 \text { ) e individuos } \\
\text { control }(n=50)\end{array}$ & $\begin{array}{l}\text { - TMA } \\
\text { - CEM } \\
\text { - EECD-IV } \\
\text { - IDB-II } \\
\text { - Escala de Actitu- } \\
\text { des Disfuncionales } \\
\text { - Índice Leiden de } \\
\text { la Sensibilidad a la } \\
\text { Depresión Revisada } \\
\text { - Escala de Clasifi- } \\
\text { cación del Humor }\end{array}$ & $\begin{array}{l}\text { RAE Positivos y } \\
\text { Negativos }\end{array}$ & $\begin{array}{l}\text { Bajo un esta- } \\
\text { do de ánimo } \\
\text { descendido, los } \\
\text { individuos con } \\
\text { antecedentes } \\
\text { de depresión } \\
\text { presentaron re- } \\
\text { cuerdos positivos } \\
\text { menos vividos }\end{array}$ \\
\hline 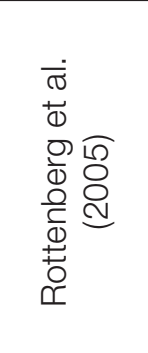 & $\begin{array}{l}\text { Pacientes } \\
\text { externados con } \\
\text { depresión mayor } \\
(n=26)\end{array}$ & $\begin{array}{l}\text { - Entrevista ideo- } \\
\text { gráfica } \\
\text { - FECD-IV } \\
\text { - ECDH } \\
\text { - Escala del Funcio- } \\
\text { namiento Global }\end{array}$ & $\begin{array}{l}\text { RAE de Valen- } \\
\text { cia Positiva y } \\
\text { Negativa }\end{array}$ & $\begin{array}{l}\text { Los RAE nega- } \\
\text { tivos con baja } \\
\text { intensidad emo- } \\
\text { cional predijeron } \\
\text { la presencia de } \\
\text { sintomatología } \\
\text { depresiva al año } \\
\text { de seguimiento }\end{array}$ \\
\hline 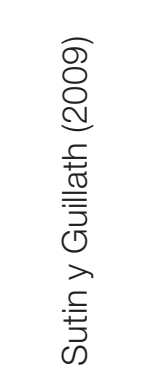 & $\begin{array}{l}\text { Estudio 1: Estu- } \\
\text { diantes universi- } \\
\text { tarios ( } n=441, \\
282 \text { mujeres) } \\
\text { Estudio 2: } \\
\text { Estudiantes uni- } \\
\text { versitarios ( } \mathrm{n}= \\
534,331 \text { mujeres) }\end{array}$ & $\begin{array}{l}\text { - CEM } \\
\text { - Programa del Afec- } \\
\text { to positivo y Negativo } \\
\text { - Inventario de las } \\
\text { Experiencias Cer- } \\
\text { canas } \\
\text { - Cuestionario Mini } \\
\text { del Humor y la An- } \\
\text { siedad }\end{array}$ & $\begin{array}{l}\text { RAE para even- } \\
\text { tos románticos, } \\
\text { coherencia e } \\
\text { intensidad emo- } \\
\text { cional }\end{array}$ & $\begin{array}{l}\text { La coherencia de } \\
\text { los RAE y la inten- } \\
\text { sidad emocional } \\
\text { medió la asocia- } \\
\text { ción entre el estilo } \\
\text { de apego evitati- } \\
\text { vo y la presencia } \\
\text { de síntomas } \\
\text { depresivos }\end{array}$ \\
\hline
\end{tabular}

EECD-IV= Entrevista Estructurada Clínica del DSM-IV. IDB-II= Inventario de Depresión de Beck - Segunda Edición. CEM= Cuestionario de la Experiencia de la Memoria. ECDH= Escala de Clasificación de la Depresión de Hamilton

Un estudio similar de Bergouignan et al. (2008) evaluó el grado de conciencia autonoética y la perspectiva visual en pacientes recuperados de un episodio depresivo e individuos control. Los resultados mostraron diferencias significativas entre ambas muestras; el grupo con antecedentes depresivos mostró una tendencia a evocar RAE de valencia positiva desde una perspectiva de tercera persona. No se encontraron diferencias en el grado de conciencia autonoética reportado. 
Por otra parte, Kuyken y Howell (2006) evaluaron los RAE en adolescentes con y sin depresión así como posibles asociaciones con antecedentes de trauma. En comparación con el grupo control, los recuerdos del grupo clínico fueron evocados prevalentemente desde una perspectiva de tercera persona, presentaron mayor importancia personal y menor antigüedad. Entre aquellos adolescentes con depresión que además reportaron antecedentes de situaciones traumáticas, los recuerdos presentaron un mayor grado de vivacidad y mayor frecuencia de evocación en comparación con aquellos adolescentes con depresión pero sin antecedentes de trauma.

En el contexto de un estudio aleatorizado de 3 meses que buscó comparar la terapia cognitiva basada en el mindfulness versus un tratamiento antidepresivo de mantenimiento (Kuyken \& Moulds, 2009), se evaluó los RAE de individuos con antecedentes depresivos al tiempo basal y al finalizar el tratamiento. Al inicio del mismo, los RAE evocados desde una perspectiva de tercera persona presentaron menor vivacidad, mayor antigüedad y fueron evocados con mayor frecuencia en comparación con los RAE recordados desde una perspectiva de primera persona. A su vez, la tendencia a evocar los RAE desde una perspectiva de tercera persona mostró asociación positiva con una evaluación negativa del sí mismo, menor disposición a los procesos de mindfulness y mayor tendencia a presentar un tipo de comportamiento evitativo. De la misma manera, los participantes que evocaron los RAE desde una perspectiva de primera persona al inicio del tratamiento presentaron menores niveles de depresión al finalizar el tratamiento. Los resultados no presentaron modificaciones aun controlando los análisis de acuerdo con la severidad de la depresión y rasgos de rumiación de los participantes. Sin embargo, no se encontraron asociaciones entre la perspectiva visual y el grado de rumiación. Los autores proponen que los individuos con depresión tienden a utilizar la perspectiva de tercera persona como una vía para tomar distancia de su pasado evitando el acceso a los recuerdos de un modo específico y detallado (Kuyken \& Moulds, 2009). 
Lolich I Psicodebate, 14(2) I 59-82.

Tabla 2.

Conciencia Autonoética y perspectiva visual.

\begin{tabular}{|c|c|c|c|c|}
\hline Estudio & $\begin{array}{c}\text { Características } \\
\text { de la Muestra }\end{array}$ & Instrumentos & $\begin{array}{l}\text { Componentes } \\
\text { fenomenológi- } \\
\text { cos bajo estudio }\end{array}$ & $\begin{array}{l}\text { Hallazgos } \\
\text { Principales }\end{array}$ \\
\hline 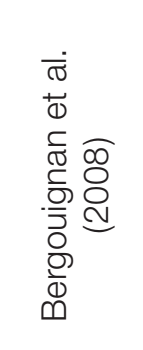 & $\begin{array}{l}\text { Individuos recupe- } \\
\text { rados de un epi- } \\
\text { sodio depresivo } \\
\text { mayor ( } n=20,17 \\
\text { mujeres) e indivi- } \\
\text { duos control ( } n= \\
20,17 \text { mujeres) }\end{array}$ & $\begin{array}{l}\text { - TMAP } \\
\text { - Entrevista Inter- } \\
\text { nacional Neurop- } \\
\text { siquiátrica Mini } \\
\text { - ECMA }\end{array}$ & $\begin{array}{c}\text { Conciencia Auto- } \\
\text { noética y pers- } \\
\text { pectiva visual }\end{array}$ & $\begin{array}{l}\text { Los individuos } \\
\text { recuperados de } \\
\text { un episodio evo- } \\
\text { caron recuerdos } \\
\text { de valencia po- } \\
\text { sitiva desde una } \\
\text { perspectiva de } \\
\text { tercera persona }\end{array}$ \\
\hline 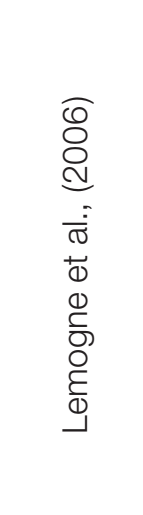 & $\begin{array}{l}\text { Individuos Depri- } \\
\text { midos } \\
\text { ( } \mathrm{n}=21 ; 13 \text { muje- } \\
\text { res) e individuos } \\
\text { control ( } \mathrm{n}=21 ; 13 \\
\text { mujeres) }\end{array}$ & $\begin{array}{l}\text { - Entrevista Inter- } \\
\text { nacional Neurop- } \\
\text { siquiátrica Mini } \\
\text { - FECD-IV } \\
\text { - ECMA } \\
\text { - IDB } \\
\text { - TMAP } \\
\text { - Procedimiento } \\
\text { Recordar/Saber } \\
\text { - Procedimiento } \\
\text { Campo/Obser- } \\
\text { vador }\end{array}$ & $\begin{array}{l}\text { Recuerdos Posi- } \\
\text { tivos y Negativos; } \\
\text { Conciencia Auto- } \\
\text { noética; Perspec- } \\
\text { tiva Visual }\end{array}$ & $\begin{array}{l}\text { Los recuerdos } \\
\text { positivos de los } \\
\text { individuos depri- } \\
\text { midos mostraron } \\
\text { menor concien- } \\
\text { cia autonoética }\end{array}$ \\
\hline 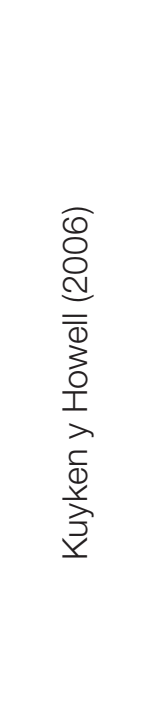 & $\begin{array}{l}\text { Adolescentes } \\
\text { deprimidos }(n= \\
31,29 \text { mujeres) } \\
\text { y adolescentes } \\
\text { sin antecedentes } \\
\text { depresivos ( } \mathrm{n}= \\
34,22 \text { mujeres) }\end{array}$ & $\begin{array}{l}\text { - FECD-IV } \\
\text { - IDB-II } \\
\text { - Cuestionario de } \\
\text { Historia Traumática } \\
\text { - Escala del Im- } \\
\text { pacto de Eventos } \\
\text { en Niños } \\
\text { - Tarea de Fluen- } \\
\text { cia verbal } \\
\text { - TMA } \\
\text { - Escala de Likert } \\
\text { sobre Dimensio- } \\
\text { nes de la Memo- } \\
\text { ria Autobiográfica } \\
\text { - Tarea de los Re- } \\
\text { cuerdos caracterís- } \\
\text { ticos del sí mismos }\end{array}$ & $\begin{array}{l}\text { Componentes } \\
\text { fenomenológicos } \\
\text { de los recuerdos }\end{array}$ & $\begin{array}{l}\text { Los adolescen- } \\
\text { tes deprimidos } \\
\text { presentaron } \\
\text { recuerdos más } \\
\text { significativos, más } \\
\text { recientes y desde } \\
\text { una perspectiva } \\
\text { de tercera perso- } \\
\text { na. Los recuerdos } \\
\text { negativos fueron } \\
\text { recordados con } \\
\text { mayor frecuencia. }\end{array}$ \\
\hline
\end{tabular}


Conciencia Autonoética y perspectiva visual. (continuación)

\begin{tabular}{|c|c|c|c|c|}
\hline Estudio & $\begin{array}{l}\text { Características } \\
\text { de la Muestra }\end{array}$ & Instrumentos & $\begin{array}{c}\text { Componentes } \\
\text { fenomenológicos } \\
\text { bajo estudio }\end{array}$ & $\begin{array}{l}\text { Hallazgos } \\
\text { Principales }\end{array}$ \\
\hline 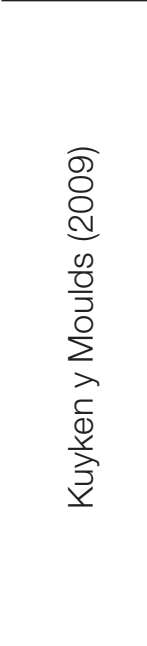 & $\begin{array}{l}123 \text { individuos } \\
\text { con anteceden- } \\
\text { tes de depresión } \\
\text { recurrente ( } 76.4 \% \\
\text { mujeres) }\end{array}$ & $\begin{array}{l}\text { - TMA } \\
\text { - ECDH } \\
\text { - FECD-IV } \\
\text { - Lista de Estados } \\
\text { Depresivos } \\
\text { - Cuestionario de } \\
\text { Rumiación-Re- } \\
\text { flexión } \\
\text { - Inventario de } \\
\text { Kentucky sobre } \\
\text { habilidades para } \\
\text { el Mindfulness } \\
\text { - Escala de Evi- } \\
\text { tación Cognitiva- } \\
\text { Comportamental }\end{array}$ & $\begin{array}{c}\text { Perspectiva visual } \\
\text { de evocación }\end{array}$ & $\begin{array}{l}\text { La perspectiva } \\
\text { de tercera perso- } \\
\text { na se encontró } \\
\text { asociada con } \\
\text { una autoevalua- } \\
\text { ción negativa } \\
\text { y conductas } \\
\text { evitativas. }\end{array}$ \\
\hline
\end{tabular}

$\mathrm{ECMA}=$ Escala de Clasificación de Montgomery y Asberg. TMAP = Tarea de la Memoria Autobiográfica del Pasado.

\section{Recuerdos Intrusivos}

Se entiende que un recuerdo se torna intrusivo cuando la evocación de eventos recientes o lejanos, usualmente de valencia negativa, se introduce en la conciencia de modo involuntario. Dicha intromisión suele ir acompañada por el afecto de la vivencia original, interfiriendo en las actividades diarias, promoviendo a su vez estados anímicos displacenteros (Meiser-Stedman et al., 2012). Evidencia previa sugiere que los individuos con depresión son particularmente propensos a experimentar RAE de tipo intrusivo (e.g. Newby \& Moulds, 2011a; Patel et al., 2007; Williams et al., 2007).

En un estudio con estudiantes universitarios que presentaban sintomatología depresiva clínicamente significativa (tabla 3), Williams y Moulds (2007) encontraron que los RAE intrusivos no sólo se caracterizaron por emociones negativas intensas sino también por la manifestación repetida de componentes sensoriales y una sensación de actualidad. Es más, dichos componentes dieron cuenta de la varianza asociada a la predicción de la severidad de los síntomas depresivos. Las referencias sensoriales fueron de carácter predominantemente visual, táctil y auditivo, si bien algunos participantes también señalaron componentes cinéticos y olfativos. En una submuestra de individuos disfóricos, 
la sensación de actualidad se mostró asociada con los niveles de depresión. Dicha asociación dio cuenta de la varianza en la predicción de los síntomas depresivos, aun controlando por el grado de severidad y frecuencia de los fenómenos intrusivos (Williams \& Moulds, 2007a).

En un estudio análogo, con estudiantes de secundario, Meiser-Stedman et al. (2012) evaluaron características de los RAE intrusivos con relación a un evento negativo reciente. La frecuencia de los RAE intrusivos, su afecto y otras características fenomenológicas explicaron la varianza de la severidad de síntomas depresivos bajo un modelo de regresión lineal. Los autores sostienen que los RAE intrusivos facilitarían la persistencia del estado de ánimo disminuido en adolescentes con sintomatología depresiva (Meiser-Stedman et al., 2012).

A su vez, Patel et al. (2007) compararon la presencia de imágenes intrusivas aisladas versus RAE de tipo intrusivo en pacientes con depresión mayor. Aquellos individuos que actualmente se encontraban transitando un episodio depresivo reportaron experimentar de modo predominante RAE de tipo intrusivo, los cuales fueron evocados con una sensación de actualidad junto con componentes afectivos y sensoriales. Además, los RAE contenían variados detalles contextuales y narrativos que resultaron más elaborados y de mayor antigüedad que las imágenes por sí solas (Patel et al., 2007). Posteriormente, a una submuestra de participantes se les administró la terapia de resignificación de los componentes fenomenológicos, orientada a reducir la frecuencia y prominencia experiencial de dichas intrusiones (Brewin et al., 2009). Se observó que los recuerdos abordados terapéuticamente fueron remplazados por nuevos recuerdos intrusivos (Patel et al., 2007).

Bajo la hipótesis de que los RAE intrusivos podrían asumir rasgos característicos durante la presencia de sintomatología depresiva, Newby y Moulds (2011a) compararon sus características en individuos: (i) actualmente deprimidos, (ii) recuperados de un episodio depresivo, y (iii) sin antecedentes afectivos. La frecuencia de los RAE de tipo intrusivo resultó similar en los tres grupos. Tampoco se encontraron diferencias en los porcentajes de pensamientos e imágenes asociadas. Sin embargo, los RAE de tipo intrusivo provenientes del grupo con depresión presentaron mayor cantidad de referencias sensoriales (predominantemente de tipo visual), elicitaron mayores niveles de estrés, de interferencias en las actividades cotidianas y fueron acompañados por mayores niveles de afecto negativo y conductas evitativas en comparación con lo reportado por los otros dos grupos. Estos mismos resultados se mantuvieron constantes aun cuando aquellos individuos sin antecedentes afectivos que sí reportaron otro tipo de sintomatología psiquiátrica fueron excluidos. Del mismo modo, el grupo con antecedentes afectivos presentó puntuaciones intermedias entre el grupo actualmente deprimido y el grupo sin antecedentes. Esto se observó 
particularmente en los componentes relativos a la vivacidad y al afecto negativo. Los autores sugieren que el modo de manifestación de dichos componentes fenomenológicos permite diferenciar a los grupos de individuos deprimidos, recuperados e individuos sin antecedentes afectivos (Newby \& Moulds, 2011a). Por otro lado, en contraposición a las hipótesis planteadas en el estudio y a trabajos previos (Kuyken \& Moulds, 2009; Lemogne et al., 2006; Williams \& Moulds, 2007b), no se encontraron diferencias con relación a los reportes relativos a la perspectiva visual entre los grupos (Newby \& Moulds, 2011a).

En otra investigación, Newby y Moulds (2011b) evaluaron en un grupo de voluntarios adultos de la población general las características de los RAE negativos de tipo intrusivos con relación a la posibilidad de que las mismas permitieran predecir la presencia de depresión a mediano plazo (seis meses). Al finalizar el seguimiento, el grado de intrusividad, de impacto emocional, de descontrol percibido y de interferencia de los RAE en la vida diaria resultaron significativos como variables predictoras de la presencia de sintomatología depresiva a los 6 meses de seguimiento (Newby \& Moulds, 2011b).

Del mismo modo que sucede con los recuerdos intrusivos relativos a eventos significativos, las experiencias negativas de tipo traumáticas conforman puntos vitales de referencia personal. Newby y Moulds (2011c) evaluaron las características de eventos negativos recientes en individuos con depresión mayor, individuos recuperados de uno o más episodios previos e individuos control. En un primer tiempo, los tres grupos no mostraron diferencias en cuanto a la apreciación del grado de centralidad del recuerdo. Seis meses más tarde, los síntomas depresivos fueron reevaluados. Los resultados indicaron la ausencia de asociaciones significativas entre la centralidad del evento y la presencia de sintomatología afectiva, ya sea en el momento inicial de evaluación o a posteriori. Sin embargo, como se esperaba, los puntajes relativos a la centralidad se encontraron positivamente asociados con procesos de intrusión y rumiación del recuerdo. 
Lolich I Psicodebate, 14(2) I 59-82.

Tabla 3.

Componentes Fenomenológicos de los Recuerdos Intrusivos.

\begin{tabular}{|c|c|c|c|c|}
\hline Estudio & $\begin{array}{c}\text { Características } \\
\text { de la Muestra }\end{array}$ & Instrumentos & $\begin{array}{l}\text { Componentes } \\
\text { fenomenológi- } \\
\text { cos bajo estudio }\end{array}$ & $\begin{array}{l}\text { Hallazgos Prin- } \\
\text { cipales }\end{array}$ \\
\hline 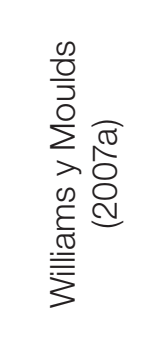 & $\begin{array}{c}70 \text { estudiantes } \\
\text { con depresión y } \\
250 \text { estudiantes } \\
\text { sin patología (168 } \\
\text { mujeres) }\end{array}$ & $\begin{array}{l}\text { - FECD-IV } \\
\text { - Entrevista de } \\
\text { los Recuerdos } \\
\text { Intrusivos } \\
\text { - IDB-II } \\
\text { - Escala de Admi- } \\
\text { nistración Clínica } \\
\text { Post-Trauma }\end{array}$ & $\begin{array}{c}\text { Características } \\
\text { fenomenológicas } \\
\text { de los recuerdos } \\
\text { intrusivos }\end{array}$ & $\begin{array}{l}\text { Los componentes } \\
\text { sensoriales predi- } \\
\text { jeron la severidad } \\
\text { de los síntomas } \\
\text { depresivos }\end{array}$ \\
\hline 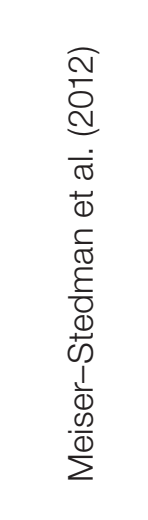 & $\begin{array}{c}\text { Estudiantes de se- } \\
\text { cundario ( } n=231 \\
146 \text { mujeres) }\end{array}$ & $\begin{array}{l}\text { - Escala Revisada } \\
\text { del Impacto de } \\
\text { Eventos en Niños } \\
\text { - Escala de Cla- } \\
\text { sificación Auto- } \\
\text { Administrable de } \\
\text { la Depresión de } \\
\text { Birleson } \\
\text { - Cuestionario de } \\
\text { la Cualidades de } \\
\text { los Recuerdos } \\
\text { Traumáticos }\end{array}$ & $\begin{array}{l}\text { Recuerdos intru- } \\
\text { sivos }\end{array}$ & $\begin{array}{l}\text { Las caracte- } \\
\text { rísticas de los } \\
\text { recuerdos intru- } \\
\text { sivos se encon- } \\
\text { traron asociados } \\
\text { positivamente } \\
\text { con la presencia } \\
\text { de sintomatología } \\
\text { depresiva }\end{array}$ \\
\hline 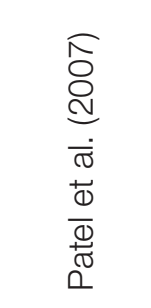 & $\begin{array}{c}\text { Individuos con } \\
\text { depresión ( } \mathrm{n}=39 \\
26 \text { mujeres) }\end{array}$ & $\begin{array}{l}\cdot \text { IDB } \\
\cdot \text { IAB } \\
\cdot \text { EED }\end{array}$ & $\begin{array}{l}\text { Características } \\
\text { fenomenológicas } \\
\text { de los recuer- } \\
\text { dos e imágenes } \\
\text { intrusivas }\end{array}$ & $\begin{array}{c}\text { La sensación } \\
\text { de actualidad, } \\
\text { las sensaciones } \\
\text { físicas y afectivas } \\
\text { caracterizaron } \\
\text { a los recuerdos } \\
\text { intrusivos }\end{array}$ \\
\hline 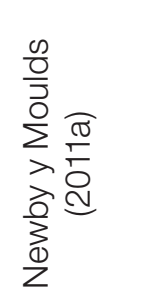 & $\begin{array}{l}\text { Individuos con } \\
\text { depresión ( } \mathrm{n}= \\
25) \text {, individuos re- } \\
\text { cuperados de un } \\
\text { episodio ( } \mathrm{n}=30 \text { ) e } \\
\text { individuos control } \\
(\mathrm{n}=30)\end{array}$ & $\begin{array}{l}\text { - FECD-IV } \\
\text { - IDB-II } \\
\text { - ERI } \\
\text { - CEM } \\
\text { - IAB } \\
\text { - EIE }\end{array}$ & $\begin{array}{c}\text { Características } \\
\text { fenomenológicas } \\
\text { de los recuerdos } \\
\text { intrusivos }\end{array}$ & $\begin{array}{l}\text { Los individuos } \\
\text { con depresión } \\
\text { reportaron mayor } \\
\text { cantidad de re- } \\
\text { cuerdos intrusivos } \\
\text { negativos y con } \\
\text { mayor vivacidad }\end{array}$ \\
\hline
\end{tabular}


Fenomenología de los Recuerdos Autobiográficos en el Espectro Depresivo: Una Revisión

Componentes Fenomenológicos de los Recuerdos Intrusivos. (continuación)

\begin{tabular}{|c|c|c|c|c|}
\hline Estudio & $\begin{array}{l}\text { Características } \\
\text { de la Muestra }\end{array}$ & Instrumentos & $\begin{array}{l}\text { Componentes } \\
\text { fenomenológi- } \\
\text { cos bajo estudio }\end{array}$ & $\begin{array}{l}\text { Hallazgos Prin- } \\
\text { cipales }\end{array}$ \\
\hline $\begin{array}{l}\frac{0}{0} \\
\frac{0}{2} \\
\stackrel{0}{0} \\
\frac{0}{0} \\
\frac{0}{2} \\
\sum^{0} \\
\lambda \\
\frac{0}{2} \\
\sum_{0}^{0} \\
\frac{1}{2}\end{array}$ & $\begin{array}{c}\text { Tiempo 1: } \\
\text { Voluntarios de la } \\
\text { comunidad ( } \mathrm{n}= \\
121) \\
\text { Tiempo 2: } \\
\text { Voluntarios de la } \\
\text { comunidad ( } \mathrm{n}= \\
\text { 64, } 33 \text { recibieron } \\
\text { tratamiento) }\end{array}$ & $\begin{array}{l}\cdot \text { IDB-II } \\
\cdot \text { IAB } \\
\cdot \text { ERI } \\
\cdot \text { CEM } \\
\cdot \text { EIE }\end{array}$ & $\begin{array}{l}\text { Componentes } \\
\text { fenomenológicos } \\
\text { de los recuerdos } \\
\text { intrusivos }\end{array}$ & $\begin{array}{c}\text { El impacto emo- } \\
\text { cional y la falta de } \\
\text { control perci- } \\
\text { bido predijeron } \\
\text { la presencia de } \\
\text { sintomatología } \\
\text { depresiva }\end{array}$ \\
\hline $\begin{array}{l}\frac{0}{0} \\
\frac{0}{2} \\
\frac{1}{0} \\
\frac{0}{0} \\
\frac{0}{2} \\
\sum^{0} \\
\lambda \\
\frac{1}{0} \\
\sum_{0}^{0} \\
\frac{0}{2}\end{array}$ & $\begin{array}{c}\text { Estudio 1: } \\
\text { individuos depri- } \\
\text { midos } \\
(\mathrm{n}=85 ; 56 \text { mu- } \\
\text { jeres); individuos } \\
\text { recuperados de } \\
\text { un episodio ( } \mathrm{n}= \\
25) \text {, individuos } \\
\text { control }(\mathrm{n}=30) \\
\text { Estudio } 2: \\
64 \text { participantes } \\
(\mathrm{n}=33 \text { recibieron } \\
\text { tratamiento) }\end{array}$ & $\begin{array}{l}\cdot \text { FECD-IV } \\
\cdot \text { IDB-II } \\
\cdot \text { IAB } \\
\cdot \text { ERI } \\
\cdot \text { CEM } \\
\cdot \text { ECE-7 } \\
\cdot \text { ERI-RSD }\end{array}$ & $\begin{array}{l}\text { Recuerdos intru- } \\
\text { sivos y centrali- } \\
\text { dad del evento }\end{array}$ & $\begin{array}{c}\text { Los puntajes de } \\
\text { centralidad se en- } \\
\text { contraron asocia- } \\
\text { dos positivamente } \\
\text { con la intrusividad } \\
\text { y los procesos de } \\
\text { rumiación }\end{array}$ \\
\hline
\end{tabular}

$\mathrm{IDB}=$ Inventario de Ansiedad de Beck. EED = Escala de Experiencias Disociativas. ERI= Entrevista de Recuerdos Intrusivos. EIE = Escala de Impacto de los Eventos. ERI-RSD= Evitación de Recuerdos Intrusivos: Rumiación, Supresión y Distracción. ECE-7= Escala de la Centralidad del Evento-7.

\section{Discusión}

Tomadas en conjunto, y si bien preliminares, las investigaciones analizadas sugieren que los RAE asumen perfiles de evocación característicos durante la presencia de alteraciones del espectro depresivo. Los estudios abordados encontraron diferencias significativas entre los individuos con sintomatología depresiva y el grupo control en las dimensiones afectividad, perspectiva visual y conciencia autonoética reportada. De la misma manera, se encontraron diferencias entre los grupos al evaluar las características fenomenológicas presentes en recuerdos de tipo intrusivo. 
Las investigaciones coincidieron en que los recuerdos de valencia positiva, en los grupos clínicos, fueron evocados desde una perspectiva visual de tercera persona (Bergouignan et al., 2008; Lemogne et al., 2006), con bajos niveles de intensidad emocional, vivacidad (Werner-Seidler \& Moulds, 2011a) y conciencia autonoética (Lemogne et al., 2006). Resulta interesante el hecho de que el estado de ánimo al momento de la evaluación se mostrara asociado a perfiles de evocación distintivos. Por ejemplo, Werner-Seidler y Moulds, (2011a) no observaron diferencias entre los grupos cuando los individuos se encontraban en un estado de ánimo neutro. En cambio, cuando ambos grupos fueron evaluados en un estado de ánimo descendido, el grupo clínico mostró evocaciones más generalizadas que el grupo control. La presencia de malestar afectivo disminuiría el acceso cognitivo a la fenomenología del recuerdo. Particularmente se vieron afectados los detalles vinculados a la vivacidad de la evocación. Diversos mecanismos cognitivos explicarían las alteraciones relativas a la evocación de recuerdos de valencia positiva. Durante la presencia de un estado de ánimo descendido, los autores proponen que se reduciría la accesibilidad a los recuerdos de un modo concreto. La evocación de eventos agradables podría generar sensaciones disruptivas cuando se transita un episodio depresivo. Estas serían la resultante de un efecto de comparación entre el momento actual con momentos de bienestar previos, por lo que se evitaría su recuerdo (Werner-Seidler \& Moulds, 2011a). En concordancia, tanto Sutin y Gillath (2009), y Rottenberg et al. (2005) señalaron que bajos niveles de afectividad y la presencia de conductas evitativas se encontraron asociados a la presencia de sintomatología depresiva. Trabajar las conductas de afrontamiento y promover un procesamiento específico de la información mnémica resultaría de utilidad para individuos con este tipo de sintomatología.

En cuanto a la perspectiva visual de evocación, los distintos trabajos analizados (Bergouignan et al., 2008; Kuyken \& Howel, 2006; Kuyken \& Moulds, 2009; Lemogne et al., 2006) coincidieron en que los individuos con depresión presentaron tendencias a evocar sus recuerdos desde una perspectiva de tercera persona. Al igual que los estudios que abordaron las deficiencias en el acceso a recuerdos de valencia positiva (Werner-Seidler \& Moulds, 2011a), los autores plantean que la asunción de una perspectiva de tercera persona resulta asociada a la presencia de mecanismos cognitivos de evitación. Se entiende que una perspectiva visual de tercera persona favorece una mayor distancia emocional de evocación.

Por su parte Kuyken y Howel (2006), Kuyken y Moulds (2009), y Lemogne et al. (2006), encontraron resultados similares en cuanto al déficit presentado por los grupos clínicos en la dimensión relativa a la conciencia autonoética del recuerdo. No obstante, y a diferencia de los otros estudios, Bergouignan et al. (2008), no encontraron diferencias entre individuos recuperados de un 
episodio y un grupo control en cuanto a los niveles de conciencia autonoética reportados. En este estudio, si bien el grupo con sintomatología depresiva evocó sus recuerdos desde una perspectiva de tercera persona, el grado de conciencia autonoética fue el mismo entre los grupos. Puede plantearse que, a diferencia de los otros estudios analizados en este trabajo (Kuyken \& Howel, 2006; Kuyken \& Moulds, 2009; Lemogne et al., 2006), los autores incluyeron muestras que se encontraban asintomáticas al momento de participar del estudio. Sería interesante en futuros trabajos evaluar si el grado de conciencia autonoética sólo se encuentra disminuido cuando se presenta sintomatología depresiva o si esta dimensión también se encuentra alterada cuando los individuos se encuentran eutímicos. Del mismo modo, podría pensarse que el tipo de perspectiva visual de tercera persona es característico de individuos con sintomatología depresiva, independientemente de si se encuentran recuperados o no del episodio afectivo.

A su vez, las características fenomenológicas reportadas en los recuerdos intrusivos permitieron diferenciar a un grupo de individuos deprimidos, de un grupo recuperado y de un grupo control. El grupo clínico evocó recuerdos más vívidos y con niveles emocionales más altos, acompañados por sensaciones de actualidad (Newby \& Moulds, 2011a; Patel et al., 2007; Williams \& Moulds, 2007). Los estudios que abordaron este tipo de recuerdos encontraron además que las características de evocación de los componentes fenomenológicos vinculados a la sensorialidad, a la intensidad afectiva y a la frecuencia de evocación, se mostraron asociados con la severidad y evolución de la sintomatología (Meiser-Stedman et al., 2012; Newby \& Moulds, 2011b; Williams \& Moulds, 2007). Estos resultados podrían ser de utilidad al momento de evaluar la presencia de recuerdos intrusivos en pacientes con sintomatología depresiva. Atender a dichos atributos de los RAE podría evitar un agravamiento del cuadro. En este sentido, y en forma complementaria, Newby y Moulds (2011c) señalaron que los recuerdos intrusivos que resultan centrales en la identidad de los individuos se mostraron asociados a mayores niveles de rumiación. Atender al perfil fenomenológico de los recuerdos puede resultar relevante al momento de buscar influir en la evolución clínica de los pacientes.

Varias limitaciones deben ser señaladas. Tal como sostiene Rottenberg et al. (2005), la mayoría de los estudios utilizaron de modo predominante un único método para elicitar la evocación de los RAE, el TMA (ver tablas 1, 2 y 3). Esta dependencia podría desorientar los esfuerzos en pos de la comprensión del funcionamiento de la MA durante la ocurrencia de sintomatología afectiva. De hecho, otros autores dentro del área de estudio de memoria han sugerido que el TMA podría ser sensible a la severidad de la sintomatología depresiva (Phillips \& Williams, 1997).

Por otro lado las palabras clave, seleccionadas a priori para ser utilizadas como disparador de la evocación de RAE, podrían resultar irrelevantes para los intereses 
de los individuos. Esto posibilita que sean evocados recuerdos autobiográficos no significativos o de tipo generalizados dado el poco involucramiento personal con la tarea (Newby \& Moulds, 2011c; Rottenberg et al., 2005). Hasta ahora, sólo unos pocos trabajos han evaluado los componentes fenomenológicos en RAE en individuos con sintomatología depresiva sin utilizar como incentivo palabras seleccionadas a priori (Newby \& Moulds, 2011c; Rottenberg et al., 2005). Futuros estudios se beneficiarían de emplear diferentes procedimientos para estimular la evocación de RAE.

En consonancia, nuevas investigaciones que busquen replicar los resultados a lo largo de diferentes tipos de RAE son necesarias (e.g. recuerdos traumáticos, recuerdos exclusivos de la primera infancia). Se podría así establecer la fuerza y generalización de los resultados (Werner-Seidler \& Moulds, 2011a). Por último, algunos estudios no incluyeron un grupo control en sus análisis (Patel et al., 2007; Rottenberg et al., 2005;) y sólo un estudio partió de una selección muestral probabilística (Kuyken \& Moulds, 2009).

Dado que los RAE se encuentran íntimamente vinculados a la construcción de narrativas de vida $y$, por tanto, involucrados en la emergencia y mantenimiento de un concepto positivo de sí mismo, explorar aquellas variables que podrían exacerbar o apaciguar el impacto de los recuerdos significativos resulta necesario (Newby \& Moulds, 2011c). Considerando la alta prevalencia y recurrencia de las patologías depresivas y su estrecha relación con el sí mismo, sería de utilidad continuar con la evaluación de los componentes fenomenológicos de los RAE.

En coincidencia con lo planteado en otros estudios, los individuos recuperados de episodios previos y aquellos actualmente deprimidos se podrían ver beneficiados de un entrenamiento en modos concretos de procesamiento de la información (Joormann et al., 2007; Williams, Teasdale, Segal, \& Soulsby, 2000). De hecho, los trastornos depresivos se caracterizan por la tendencia a un procesamiento generalizado (Williams et al., 2007). Otros trabajos, no incluidos en esta revisión por no cumplir con los criterios de inclusión, postulan de hecho que dicho déficit daría cuenta de las alteraciones halladas durante la evocación de RAE de valencia positiva (e.g., Bishop et al., 2004; Brown et al., 2007; Gillihan et al., 2007).

Del mismo modo distintos autores en el área también sostienen que las intervenciones psicoterapéuticas podrían buscar reducir la vivacidad de los recuerdos disruptivos por medio de la promoción de un procesamiento específico de las cualidades afectivas y sensoriales (Birch \& Davidson, 2007; Brewin et al., 1999). Tanto en recuerdos positivos como negativos comienza a ser mayor la evidencia a favor de implementar en pacientes con alteraciones del espectro depresivo técnicas de imaginería (e.g. Brewin et al., 2009), de reinscripción de los componentes ligados a la imaginería (Kandris \& Moulds, 2008) y la terapia de desensiblización (Rosas Uribe, López Ramírez, \& Jarero Mena, 2010). 
Especificar los detalles experienciales de los recuerdos podría potenciar los RAE de valencia positiva y disminuir las emociones disruptivas de los RAE de valencia negativa. Los modelos psicoterapéuticos se verían beneficiados substancialmente por estudios que repliquen estos resultados.

Si bien la fenomenología de los RAE es aún un campo relativamente nuevo de investigación, existe cierto nivel de acuerdo en que el modo en el que los individuos evocan su pasado se relaciona con cómo se sienten respecto a sí mismos en el presente y como se proyectan en el futuro (Conway, 2005). Los estudios analizados proveen evidencia a favor de que la fenomenología de la memoria, entre otras variables, constituye un aspecto relevante en la manifestación y abordaje de las alteraciones del espectro depresivo. Profundizar en los tratamientos que promueven la especificación de los aspectos fenomenológicos de los RAE de individuos con sintomatología depresiva podría resultar particularmente beneficioso. 


\section{Referencias}

Bergouignan, L., Lemogne, C., Foucher, A., Longin, E., Vistoli, D., Allilaire, J. F., \& Fossati, P. (2008). Field perspective deficit for positive memories characterizes autobiographical memory in euthymic depressed patients. Behaviour research and therapy, 46(3), 322-333. doi: 10.1016/j.brat.2007.12.007

Bishop, S. R., Lau, M., Shapiro, S., Carlson, L., Anderson, N. D., Carmody, J., ... Devins, G. (2004). Mindfulness: A proposed operational definition. Clinical Psychology: Science and Practice, 11, 230-241. doi: 10.1093/clipsy/bph077

Brown, K. W., Ryan, R. M., \& Creswell, J. D. (2007). Mindfulness: Theoretical foundations and evidence for its salutary effects. Psychological Inquiry, 18, 211-237. doi: 10.1080/10478400701598298

Birch, L. S., \& Davidson, K. M. (2007). Specificity of autobiographical memory in depressed older adults and its relationship with working memory and IQ. The British journal of clinical psychology / the British Psychological Society, 46(Pt 2), 175-186. doi: 10.1348/014466506X119944

Brewin, C. R., Reynolds, M., \& Tata, P. (1999). Autobiographical memory processes and the course of depression. Journal of abnormal psychology, 108(3), 511-517.

Brewin, C. R., Wheatley, I., Patel, T., Fearon, P., Hackmann, A., Wells, A., ... Myers, S. (2009) Imagery rescripting as a brief stand-alone treatment for depressed patients with intrusive memories. Behaviour Research and Therapy 47, 569-576. doi: 10.1016/j.brat.2009.03.008

Cohen, G. (2008). The study of everyday memory. En G. Cohen \& M. A. Conway (Eds.), Memory in the real world (3rd ed., pp. 1-20). New York: Psychology Press.

Conway, M. A. (2005). Memory and the self. Journal of Memory and Language, 53(4), 594-628. doi: 10.1016/j.jm1.2005.08.005

Conway, M. A., \& Pleydell-Pearce, C. W. (2000). The construction of autobiographical memories in the self-memory system. Psychological Review, 107, 261-268. doi: 10.1037/0033-295X.107.2.261 
Gillihan, S. J., Kessler, J., \& Farah, M. J. (2007). Memories affect mood: evidence from covert experimental assignment to positive, neutral, and negative memory recall. Acta psychologica, 125(2), 144-154. doi: 10.1016/j.brat.2007.12.007

Greenberg, D. L., \& Rubin, D. C. (2003). The neuropsychology of autobiographical memory. Cortex, 39, 687-728.

Haringsma, R., Spinhoven, P., Engels, G. I., \& van der Leeden, R. (2010). Effects of sad mood on autobiographical memory in older adults with and without lifetime depression. The British journal of clinical psychology / the British Psychological Society, 49(Pt 3), 343-357. doi: 10.1348/014466509X454840

Hermans, D., Defranc, A., Raes, F., Williams, J. M. G., \& Eelen, P. (2005). Reduced autobiographical memory specificity as an avoidant coping style. The British journal of clinical psychology / the British Psychological Society, 44(Pt 4), 583-589. doi: 10.1348/014466505X53461

Joormann, J., Siemer, M., \& Gotlib, I. H. (2007). Mood regulation in depression: Differential effects of distraction and recall of happy memories on sad mood. Journal of Abnormal Psychology, 116, 484-490. doi: 10.1037/0021843X.116.3.484

Kandris, E., \& Moulds, M. L. (2008). Can imaginal exposure reduce intrusive memories in depression? A case study. Cognitive Behaviour Therapy, 37, 216-220. doi: 10.1080/16506070802117950

Kuyken, W., \& Howell, R. (2006). Facets of autobiographical memory in adolescents with major depressive disorder and never-depressed controls. Cognition \& Emotion, 20, 466-487. doi: 10.1080/02699930500342639

Kuyken, W., \& Moulds, M. L. (2009). Remembering as an observer: how is autobiographical memory retrieval vantage perspective linked to depression? Memory, 17(6), 624-634. doi: 10.1080/09658210902984526

Lemogne, C., Piolino, P., Friszer, S., Claret, A., Girault, N., Jouvent, R., ... Fossati, P. (2006). Episodic autobiographical memory in depression: Specificity, autonoetic consciousness, and self-perspective. Consciousness and cognition, 15(2), 258-268. doi: 10.1016/j.concog.2005.07.005

Meiser-Stedman, R., Dalgleish, T., Yule, W., \& Smith, P. (2012). Intrusive memories and depression following recent non-traumatic negative life events in adolescents. Journal of affective disorders, 137(1-3), 70-78. doi: 10.1016/j. 
jad.2011.12.020

Newby, J. M., \& Moulds, M. L. (2011a). Characteristics of intrusive memories in a community sample of depressed, recovered depressed and never-depressed individuals. Behaviour research and therapy, 49(4), 234-243. doi: 10.1016/j. brat.2011.01.003

Newby, J. M., \& Moulds, M. L. (2011b). Do intrusive memory characteristics predict depression at 6 months? Memory, 19(5), 538-546. doi: $10.1080 / 09658211.2011 .590505$

Newby, J. M., \& Moulds, M. L. (2011c). Intrusive memories of negative events in depression: is the centrality of the event important? Journal of behavior therapy and experimental psychiatry, 42(3), 277-283. doi: 10.1016/j. jbtep.2010.12.011

Patel, T., Brewin, C. R., Wheatley, J., Wells, A., Fisher, P., \& Myers, S. (2007). Intrusive images and memories in major depression. Behaviour research and therapy, 45(11), 2573-2580. doi: 10.1016/j.brat.2007.06.004

Phillips, S., \& Williams, J. M. G. (1997). Cognitive impairment, depression and the specificity of autobiographical memory in the elderly. British Journal of Clinical Psychology, 36, 341-347.

Philippot, P., Schaefer, A. \& Herbette, G. (2003). Consequences of specific processing of emotional information: Impact of general versus specific autobiographical memory priming on emotion elicitation. Emotion, 3, 270-83. doi: $10.1037 / 1528-3542.3 .3 .270$

Rosas Uribe, M. E., López Ramírez, E. O. \& Jarero Mena I. (2010). Effect of the EMDR Psychotherapeutic Approach on Emotional Cognitive Processing in Patients with Depression. The Spanish journal of psychology, 13, 396-405. doi: $10.1017 / \mathrm{S} 1138741600003954$

Rottenberg, J., Joormann, J., Brozovich, F., \& Gotlib, I. H. (2005). Emotional intensity of idiographic sad memories in depression predicts symptom levels 1 year later. Emotion (Washington, D.C.), 5(2), 238-242. doi: 10.1037/15283542.5.2.238

Rubin, D. C., Schrauf, R. W., \& Greenberg, D. L. (2003). Belief and recollection of autobiographical memories. Memory \& cognition, 31(6), 887-901.

Rubin, D. C., \& Siegler, I. C. (2004). Facets of personality and the phenomenology 
of autobiographical memory. Applied Cognitive Psychology, 18(7), 913-930. doi: 10.1002/acp.1038

Segal, Z. V., Williams, J. M. G., \& Teasdale, J. D. (2002). Mindfulness-based cognitive therapy for depression: A new approach for preventing relapse. New York: Guilford Press.

Singer, J. A., \& Salovey, P. (1988). Mood and memory: Evaluating the network theory of affect. Clinical Psychology Review, 8, $211-251$.

Sutin, A. R., \& Robins, R. W. (2007). Phenomenology of autobiographical memories: the memory experiences questionnaire. Memory, 15(4), 390-411. doi: 10.1080/09658210701256654

Sutin, A. R., \& Gillath, O. (2009). Autobiographical memory phenomenology and content mediate attachment style and psychological distress. Journal of Counseling Psychology, 56(3), 351-364. doi: 10.1037/a0014917

Tulving, E. (1985). Memory and consciousness. Canadian Psychology, 26, 1-12.

Tulving, E. (2002). Episodic Memory: From Mind to Brain. Annual Review of Psychology, 53, 1-25. doi: 10.1146/annurev.psych.53.100901.135114

Werner-Seidler, A., \& Moulds, M. L. (2011a). Autobiographical memory characteristics in depression vulnerability: formerly depressed individuals recall less vivid positive memories. Cognition \& emotion, 25(6), 1087-1103. doi: 10.1080/02699931.2010.531007

Werner-Seidler, A., \& Moulds, M. L. (2011b). Mood repair and processing mode in depression. Emotion, 12(3), 470-478. doi: 10.1037/a0025984

Williams, A. D., \& Moulds, M. L. (2007a). An investigation of the cognitive and experiential features of intrusive memories in depression. Memory, 15(8), 912-920. doi: 10.1080/09658210701508369

Williams, A. D., \& Moulds, M. L. (2007b). Cognitive avoidance of intrusive memories: recall vantage perspective and associations with depression. Behaviour Research and Therapy, 45, 1145-3. doi: 10.1016/j.brat.2006.09.005

Williams, J. M. G., Barnhofer, T., Crane, C., Herman, D., Raes, F., Watkins, E., \& Dalgleish, T. (2007). Autobiographical memory specificity and emotional disorder. Psychological bulletin, 133(1), 122-48.

Williams, J. M. G. (1996). Depression and the specificity of autobiographical 
memory. In D. C. Rubin (Ed.), Remembering our past: Studies in autobiographical memory (pp. 244-267). Cambridge, England: Cambridge University Press.

Williams, J. M. G., Teasdale, J. D., Segal, Z. V., \& Soulsby, J. (2000). Mindfulnessbased cognitive therapy reduces overgeneral autobiographical memory in formerly depressed patients. Journal of Abnormal Psychology, 109, 150-155. doi: 10.1037//0021-843X.109.1.150

Williams, J. M. G., \& Broadbent, K. (1986). Autobiographical memory in suicide attempters. Journal of abnormal psychology, 95(2), 144-149.

Williams, J. M. G., \& Scott, J. (1988). Autobiographical memory in depression. Psychological medicine, 18(3), 689-695.

Williams, J. M. G., Watts, F. N., MacLeod, C., \& Mathews, A. (1988). Cognitive psychology and emotional disorders. Chichester: England-Wiley.

Zinbarg, R., \& Rekart, K. (2006). Autobiographical memory in dysphoric and non dysphoric college students using a computerised version of the AMT. Cognition \& Emotion, 20(3/4), 506-515. doi: 10.1080/02699930500341318 